\title{
Análise das áreas queimadas e das emissões dos gases do efeito estufa no Parque Estadual da Serra do Tabuleiro - Santa Catarina
}

Gabriel Pereira, Nelson Jesus Ferreira, Elisabete Caria Moraes*, Francielle da Silva Cardozo** e Saulo Ribeiro de Freitas ${ }^{* *}$

\section{Resumo}

O objetivo principal deste trabalho é analisar as queimadas no Parque Estadual da Serra do Tabuleiro - Santa Catarina (SC) e estimar a quantidade de gases do efeito estufa emitidos neste processo. Utilizaram-se imagens do sensor CCD do China-Brazil Earth-Resources Satellite (CBERS 2 e CBERS 2B) e do Thematic Mapper (TM) do Landsat 5. A partir da metodologia do Intergovernamental Panel on Climate Change (IPCC) estimou-se que em oito anos (2001 a 2008) foi exposta à queima uma área correspondente a 1273 campos de futebol, liberando para a atmosfera aproximadamente $13.000 \mathrm{t}$ de dióxido de carbono $\left(\mathrm{CO}_{2}\right)$, $525 \mathrm{t}$ de monóxido de carbono $(\mathrm{CO}), 20 \mathrm{t}$ de metano $\left(\mathrm{CH}_{4}\right), 7 \mathrm{t}$ de óxido nitroso $\left(\mathrm{N}_{2} \mathrm{O}\right)$ e $250 \mathrm{t}$ de óxido nítrico $(\mathrm{NO})+$ dióxido de azoto $\left(\mathrm{NO}_{2}\right)$. Além disto, verificou-se uma redução nos valores de albedo, reflectância no visível e no infravermelho solar na área queimada de $83 \%, 50 \%$ e $80 \%$.

Palavras-chave: Queimadas; Gases do efeito estufa; Sensoriamento remoto.

Divisão de Sensoriamento Remoto / DSR - Instituto Nacional de Pesquisas Espaciais / INPE ( \{gabriel, nelson, bete\}@dsr.inpe.br).

** PPGG - CFH/UFSC (franciellecardozo@yahoo.com.br).

*** Centro de Previsão de Tempo e Estudos Climáticos / CPTEC - Instituto Nacional de Pesquisas Espaciais / INPE (sfreitas@cptec.inpe.br).

Geosul, Florianópolis, v. 24, n. 47, p 113-130, jan./jun. 2009 
PEREIRA, G. et al. Análise das áreas queimadas e das emissões dos gases ...

Biomass burning areas and of green house gases emission analysis in Serra do Tabuleiro State Park - Santa Catarina

\begin{abstract}
The main goals of the present work are the analysis of the biomass burning and the assessment of greenhouses gases emissions released into atmosphere in Serra do Tabuleiro State Park located in the state of Santa Catarina (SC). In this article, CCD sensor on China-Brazil Earth-Resources Satellite (CBERS) 2 and CBERS 2B platforms and Thematic Mapper (TM) sensor on Landsat 5 platform were used to extract the burned area. Through Intergovernmental Panel on Climate Change (IPCC) methodology, were found in 8 years (2001 to 2008) a amount of 1273 soccer fields were exposed to burn, releasing into atmosphere approximately $13.000 \mathrm{t}$ of carbon dioxide $\left(\mathrm{CO}_{2}\right), 525 \mathrm{t}$ of carbon monoxide $(\mathrm{CO}), 20 \mathrm{t}$ of methane $\left(\mathrm{CH}_{4}\right), 7 \mathrm{t}$ of nitrous oxide $\left(\mathrm{N}_{2} \mathrm{O}\right)$ and $250 \mathrm{t}$ of nitric oxide $(\mathrm{NO})$ and nitrogen dioxide $\left(\mathrm{NO}_{2}\right)$. Moreover, the result shows a reduction of $83 \%, 50 \%$ and $80 \%$ in albedo, visible and infrared solar reflectance values in the burnt areas.

Key words: Biomass burning; Greenhouse gases; Remote sensing.

\section{Introdução}

Existem evidências de que as queimadas ocorriam nos ecossistemas terrestres desde a Era Paleozóica no final do período Siluriano, a aproximadamente 415 milhões de anos (Ma). Nesta época os níveis de oxigênio $\left(\mathrm{O}_{2}\right)$ começavam a aumentar devido ao surgimento de plantas vasculares, o que permitiu o avanço das queimadas naturais provocadas principalmente por raios e combustão espontânea. Detritos das combustões de biomassa são bem representados na Era Mesozóica, onde os fósseis encontrados em sedimentos marinhos e terrestres, em hidrocarbonetos aromáticos cíclicos e em vegetações carbonizadas remotam ao período Jurássico e Cretáceo, fato que indica a presença de paleofogos (GLASSPOOL et al., 2004; FINKELSTEIN et al., 2006; BERNER et al., 2003; SCOTT, 2000; VENKATESAN; DAHL, 1989; KILLOPS; MASSOUD, 1992; FINKELSTEIN, 2004).
\end{abstract}


PEREIRA, G. et al. Análise das áreas queimadas e das emissões dos gases ...

Nos dias atuais estima-se que aproximadamente $90 \%$ das queimadas sejam de origem antrópica (ANDREAE, 1991). A queima de biomassas vegetais vivas e/ou mortas, em condições ideais de completa combustão, produz dióxido de carbono $\left(\mathrm{CO}_{2}\right)$ e vapor d'água $\left(\mathrm{H}_{2} \mathrm{O}\right)$, de acordo com a reação a seguir (LEVINE, 1994):

$$
\mathrm{CH}_{2} \mathrm{O}+\mathrm{O}_{2} \rightarrow \mathrm{CO}_{2}+\mathrm{H}_{2} \mathrm{O}
$$

onde $\mathrm{CH}_{2} \mathrm{O}$ representa a composição média da biomassa vegetal. Porém, o processo de combustão geralmente não é completo, sendo comum encontrar, além do $\mathrm{CO}_{2}$, outras espécies de gases, entre eles, destaca-se o $\mathrm{CO}$, o $\mathrm{CH}_{4}$, o $\mathrm{NO}$, o $\mathrm{NO}_{2}$ e o cloreto de metil $\left(\mathrm{CH}_{3} \mathrm{Cl}\right)$ (BADARINATH et al., 2004).

O impacto das queimadas nos ciclos biogeoquímicos, no clima mundial, na química atmosférica, na população em geral e na biodiversidade, constitui-se numa grande preocupação mundial. Estudos indicam que as ocorrências de grandes incêndios irão aumentar significativamente nas próximas décadas (IPCC, 2000).

O Parque Estadual da Serra do Tabuleiro, criado em 1975, é afetado constantemente pelas queimadas. $\mathrm{O}$ parque reúne praticamente todos os tipos de vegetação presentes no Estado de Santa Catarina, com exceção da Floresta Estacional Decidual do rio Uruguai (OLIVEIRA, 2005), possuindo uma extrema importância física, biológica e econômica. $\mathrm{O}$ conjunto de características do parque apresenta enorme potencial para atividades científicas, educativas e turísticas.

O presente trabalho tem como objetivo principal delimitar a área queimada no Parque Estadual da Serra do Tabuleiro para os anos de 2001 a 2008, verificando sua área de atuação e de degradação ambiental. Além disso, foram calculadas as emissões dos gases do efeito estufa liberados por estas queimadas e avaliadas alterações no albedo, na reflectância na faixa espectral do visível e no infravermelho solar (infravermelho próximo e médio). Pretende-se, assim, promover uma fonte de informação para 
PEREIRA, G. et al. Análise das áreas queimadas e das emissões dos gases ...

medidas mitigadoras, que possam contribuir com a preservação da unidade de conservação do Parque Estadual da Serra do Tabuleiro.

\section{Materiais e métodos}

\section{Localização do Parque Estadual da Serra do Tabuleiro}

O Parque Estadual da Serra do Tabuleiro está localizado entre as latitudes $27^{\circ} 41^{\prime} 09^{\prime \prime} \mathrm{S}$ e $28^{\circ} 12^{\prime} 42^{\prime}$ 'S e longitudes $48^{\circ} 49^{\prime} 20^{\prime}$ 'O e $48^{\circ} 25^{\prime} 08^{\prime}$ ' $\mathrm{O}$, ocupa uma área com mais de 92.000 ha, equivalente a $1 \%$ do território do Estado de Santa Catarina. O Parque Estadual da Serra do Tabuleiro é uma das maiores unidades de conservação do sul do Brasil e está na lista dos habitats naturais críticos da região da América Latina e do Caribe (Ambiente Brasil, 2007). A área do parque abrange nove municípios, entre eles Florianópolis (menos de 1\%), Palhoça (19\%), Paulo Lopes (29\%), Garopaba (1\%), Imaruí (8\%), São Martinho (2\%), São Bonifácio (11\%), Águas Mornas (9\%) e Santo Amaro da Imperatriz (21\%), compreendendo as Serras do Tabuleiro ao norte, o morro do Cambirela a nordeste, a Serra do Morretes a leste e a Serra do Capivari ao sul (ROSÁRIO, 2003). A Figura 1 mostra o setor analisado do Parque Estadual da Serra do Tabuleiro, com o perfil A-B no sentido Nor-Nordeste (NNE) a Su-sudoeste (SSO) traçado para se adquirir os valores de albedo, reflectância no visível e a reflectância no infravermelho de uma área queimada na região da praia da Pinheira. 
PEREIRA, G. et al. Análise das áreas queimadas e das emissões dos gases ...

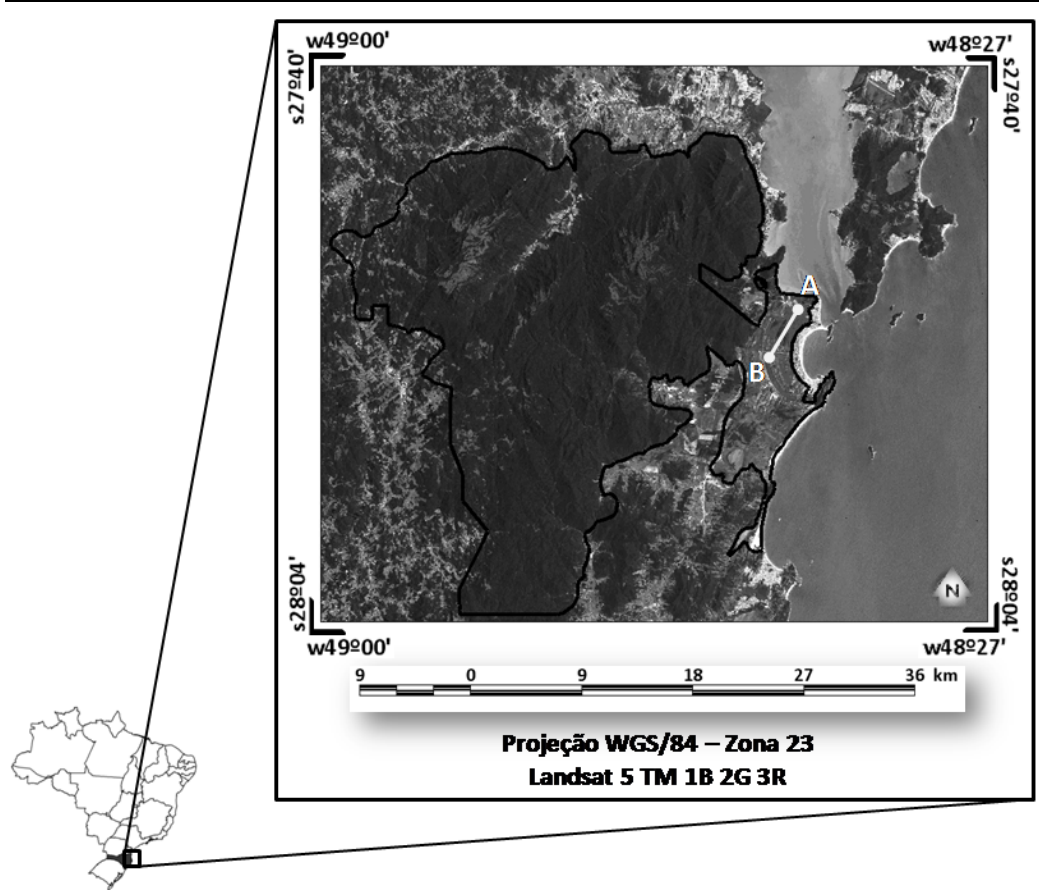

Figura 1: Localização da área de estudo inserida dentro do Parque Estadual da Serra do Tabuleiro e perfil A-B.

\section{Histórico de queimadas para Santa Catarina}

Para o estudo da distribuição espacial das queimadas foram utilizados os dados disponíveis no banco de dados de queimadas do CPTEC (http://www.cptec.inpe.br/queimadas). Estes dados representam uma série histórica de 16 anos (1992 a 2007) dos focos de queimada (hot spots) coletados pelos satélites NOAA-11, NOAA-12, NOAA-14, NOAA-15, NOAA-16, NOAA-17, NOAA18, GOES-8, GOES-12, EOS-AQUA, EOS-TERRA.

A Figura 2 mostra o total de focos acumulados para o Estado de Santa Catarina nestes 16 anos de observações. Percebe-se uma concentração de focos no centro-oeste, oeste e sudeste do Estado, 
PEREIRA, G. et al. Análise das áreas queimadas e das emissões dos gases ...

$\mathrm{Na}$ região de estudo a quantidade de focos por ponto de grade de $20 \mathrm{~km}$ varia de 4 a 10 focos.

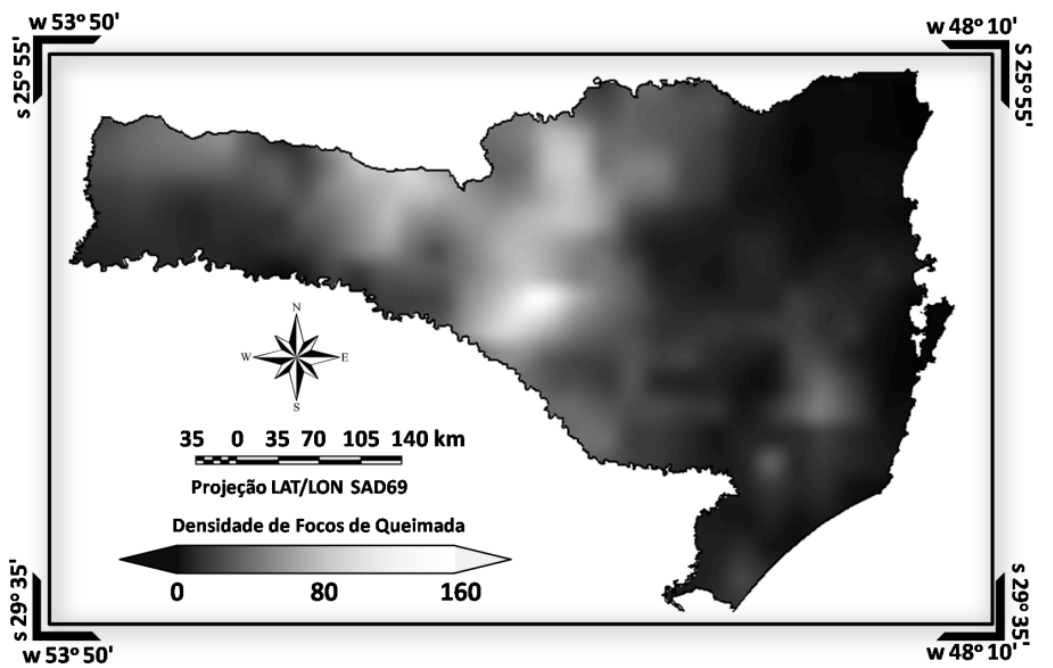

Figura 2: Densidade de focos de queimada acumulados por ponto de grade de $20 \mathrm{~km}$ para o Estado de Santa Catarina

\section{Características físicas}

O Parque Estadual da Serra do Tabuleiro está inserido dentro do domínio geomorfológico Serras do Leste Catarinense, se estendendo na direção Nordeste - Sudoeste caracterizado por uma seqüência de serras com disposição subparalela, intensa dissecação, vales profundos com encostas íngremes, sulcadas e separadas por cristas bem marcadas, possuindo pontos altimétricos com mais de 1.200 metros (MAIA, 2006). A área de estudo possui cordões arenosos semicirculares de restinga, sulcadas e separadas por cristas bem marcadas.

A cobertura vegetal agrega grande parte da diversidade da Mata Atlântica. Existem cinco tipos de vegetação no parque: a Restinga Litorânea, a Floresta Atlântica (Floresta Ombrófila Densa), Mata de Araucária (Floresta Ombrófila Mista), Vegetação 
PEREIRA, G. et al. Análise das áreas queimadas e das emissões dos gases ...

dos Campos de Altitude e Manguezal. Segundo Rosário (2003), "em todo este sistema paisagístico estão contidos, sem dúvida, habitats importantes para a preservação das espécies raras e endêmicas da floresta Atlântica", que possui aproximadamente 2.250 espécies. A fauna do parque registra 359 espécies de aves, $60 \%$ da avifauna do Estado e 76 espécies de mamíferos.

Atualmente, nos vales ocorre a substituição de florestas pela agricultura e nas encostas menos íngremes é comum encontrar reflorestamento de Pinus. A Figura 3 mostra o uso e ocupação da terra para o setor do Parque Estadual da Serra do Tabuleiro analisado. Neste Setor, que abrange os municípios de Florianópolis, Palhoça, Paulo Lopes, Garopaba, São Bonifácio, Águas Mornas e Santo Amaro da Imperatriz, a vegetação arbórea ocupa aproximadamente $720 \mathrm{~km}^{2}$, as áreas de vegetação arbustiva/campos/restinga representam $63,43 \mathrm{~km}^{2}$, as áreas de manguezal cobrem apenas $1,02 \mathrm{~km}^{2}$ e a hidrografia, composta principalmente pela Lagoa do Siriú e pelo rio da Madre, ocupam aproximadamente $9 \mathrm{~km}^{2}$. As áreas antropizadas representam aproximadamente $55 \mathrm{~km}^{2}$ de toda a cobertura, com destaque para o desmatamento que ocorre nas Serras do Tabuleiro e no morro do Cambirela com $37,15 \mathrm{~km}^{2}$, para a agricultura nas áreas mais planas com aproximadamente $14 \mathrm{~km}^{2}$ e para áreas urbanas próximas as áreas litorâneas com $3,35 \mathrm{~km}^{2}$. 
PEREIRA, G. et al. Análise das áreas queimadas e das emissões dos gases ...

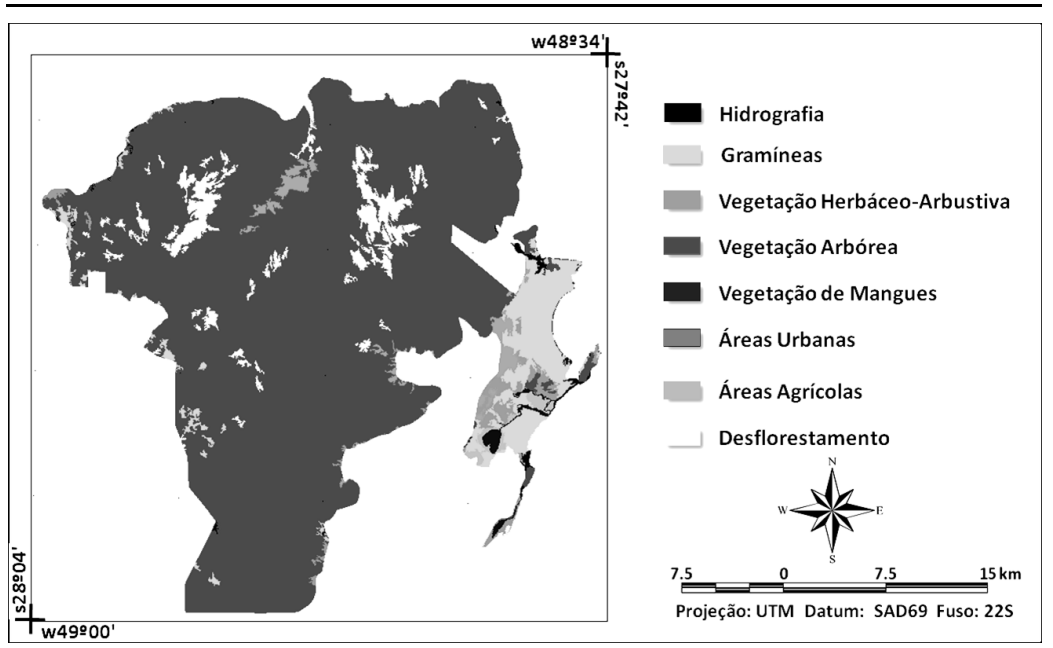

Figura 3: Uso e ocupação da terra da área de estudo inserida dentro do Parque Estadual da Serra do Tabuleiro

\section{Metodologia}

Para o presente trabalho foram utilizadas imagens do satélite Landsat 5 (L5) sensor Thematic Mapper (TM) que possui uma resolução espacial nominal de 30 metros, e capta informações espectrais em 7 bandas, referentes ao visível, infravermelho próximo, médio e distante, ambas com resolução radiométrica de 8 bits e resolução temporal de 16 dias. Além disto, utilizou-se o satélite sino-brasileiro CBERS 2 e 2B (China-Brazil Earth Resources Satellite) sensor CCD (High Resolution CCD Camera) com resolução espacial nominal de 20 metros, resolução radiométrica de 8 bits, resolução temporal de 26 dias e com 5 bandas espectrais referentes ao visível e infravermelho próximo.

Para o processamento das imagens e análise da área queimada utilizou-se o aplicativo de geoprocessamento SPRING 4.3.1 (Sistema de Processamento de Informações Georeferenciadas). Neste aplicativo, 22 imagens dos satélites Landsat 5, CBERS 2 e CBERS - 2B foram inseridas e corrigidas geometricamente a partir do modelo polinomial de $1^{\circ}$ grau e do 
PEREIRA, G. et al. Análise das áreas queimadas e das emissões dos gases ...

interpolador vizinho mais próximo. As imagens foram registradas a partir da base geocover disponibilizada pela NASA (National Aeronautics and Space Administration), originadas de dados da série Landsat, submetidos a procedimentos de ortorretificação para minimizar os deslocamentos sofridos na imagem pelo relevo. Os dados geocover são disponibilizados no formato Mr. Sid (.sid) com projeção UTM/WGS-84 (GLCF, 2004).

Para a análise do albedo e das reflectâncias espectrais nas faixas do visível e do infravermelho solar foram utilizadas imagens $\mathrm{TM} /$ Landsat 5 de 21/11/2005. Os níveis de cinza (NC) foram transformados em reflectância aparente pelo método proposto por Chander e Markham (2003). Após este procedimento utilizou-se o modelo de transferência radiativa second simulation of the satellite signal in the solar spectrum (6S) (VERMOTE, 1997) para minimizar os efeitos da interferência atmosférica no sinal captado pelo sensor orbital. Foram adotadas como condições iniciais a visibilidade de $70 \mathrm{~km}$, o modelo de aerossóis marítimo e a atmosfera tropical. Assumiu-se como principais efeitos atmosféricos a absorção pelos gases (vapor de água, dióxido de carbono, oxigênio e ozônio) e espalhamento pelas moléculas dos gases atmosféricos.

Para se obter os valores de albedo e das reflectâncias no visível e no infravermelho solar, os valores de reflectância de superfície nas bandas do TM/Landsat 5 foram convertidos a partir da fórmula proposta por Liang (2000), cujos os coeficientes foram ajustados por Pereira et al. (2007):

$$
\begin{aligned}
& \text { Albedo }=0,420 * \alpha_{1}+0,153 * \alpha_{3}+0,440 * \alpha_{4}+0,100^{*} \alpha_{5}+0,084^{*} \alpha_{7} \\
& \quad-0,0018 \\
& \text { Reflectância }_{\text {visivel }}=0,564 * \alpha_{1}+0,417^{*} \alpha_{2}+0,306^{*} \alpha_{3} \\
& \text { Reflectância }_{I V}=0,755^{*} \alpha_{4}+0,231^{*} \alpha_{5}+0,126^{*} \alpha_{7}-0,003
\end{aligned}
$$

onde $\alpha_{\mathrm{n}}$ representa a banda do TM/Landsat 5 utilizada.

Para se estimar a quantidade de gases $\mathrm{CO}_{2}, \mathrm{CO}, \mathrm{CH}_{4}, \mathrm{~N}_{2} \mathrm{O}$ e $\mathrm{NO}_{\mathrm{x}}$ emitidos para a atmosfera pela queima de biomassa, utilizouse a metodologia proposta no Revised 1996 Guidelines of 
PEREIRA, G. et al. Análise das áreas queimadas e das emissões dos gases ...

Intergovernmental Panel on Climate Change (IPCC, 1997). A metodologia consiste em quantificar a área queimada (A dada em

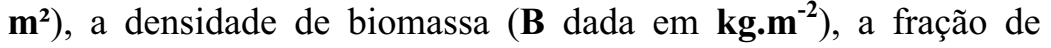
biomassa acima do solo $(\boldsymbol{\alpha})$, a eficiência de queima $(\boldsymbol{\beta})$ e o fator de emissão de gases, o qual apresenta dependência com o tipo de bioma em combustão $\left(\mathbf{E F}_{\mathbf{x}}\right.$ em $\left.\mathbf{g} \cdot \mathbf{k g}^{-1}\right)$ (SEILER; CRUTZEN, 1980), como mostra a fórmula (5).

$$
M_{x}=E F_{x} * A^{*} B * \alpha * \beta
$$

No cálculo das propriedades da biomassa, como, por exemplo, fração viva, fração viva oxidada, quantidade de carbono, eficiência de queima, quantidade de carbono da biomassa morta, entre outros, utilizaram-se os valores propostos por MCT (2002). Nestes, os valores das emissões são obtidos a partir da relação entre o total de carbono emitido, a taxa de emissão do composto relativa ao elemento e a taxa do peso molecular do mesmo composto.

\section{Resultados e discussões}

Ao analisar as áreas queimadas, percebe-se que todas as cicatrizes se encontram apenas na região da praia da Pinheira. A Figura 4 mostra as imagens $\mathrm{TM} /$ Landsat 5 para as datas de 02/09/2002 (a), 08/04/2004 (b) e 21/11/2005 (c) com as diferentes cicatrizes de queimadas encontradas para essas áreas. Segundo relatórios do corpo de bombeiros, grande parte das queimadas é de origem antropogênica e servem, entre outros objetivos, para a renovação de pastos para o gado. Nas áreas queimadas a vegetação predominante é composta principalmente por gramíneas e pequenos arbustos (fisionomia de campos e restinga). 
PEREIRA, G. et al. Análise das áreas queimadas e das emissões dos gases ...

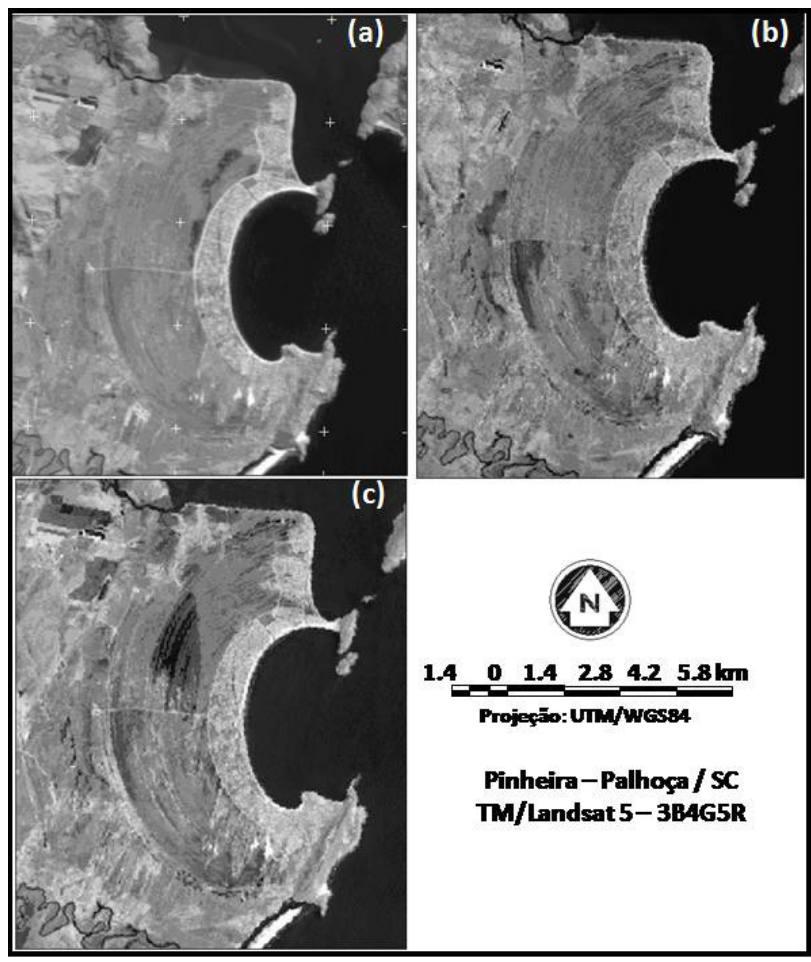

Figura 4: Imagens TM/Landsat 5 que mostram cicatrizes de queimadas para três períodos distintos.

Dadas as condições de aquisição das imagens dos sensores CCD/CBERS 2, CBERS 2B e TM/Landsat 5, como a influência de nuvens, entre outros fatores, procurou-se quantificar as áreas queimadas a partir de suas características espectrais, assim como as cicatrizes de queimadas facilmente visualizadas em imagens multiespectrais. Realizaram-se algumas verificações de campo em 2005 e 2006 para validar a classificação visual das imagens, realizadas a partir de edição topológica manual.

A estimativa da área queimada para o período de 26/05/2001 a 25/12/2008 foi de aproximadamente $13,75 \mathrm{~km}^{2}$ (Figura 5) correspondendo a $1,5 \%$ de todo o território do parque, equivalente 
PEREIRA, G. et al. Análise das áreas queimadas e das emissões dos gases ...

a 1273 campos de futebol. Com base na área queimada calculada, a estimativa da emissão de gases do efeito estufa foi feita de acordo com a metodologia do IPCC (1997) prescrita para regiões onde a vegetação predominante é de campos. Foram utilizados os valores dos parâmetros estimados por MCT (2002) e descrito na Tabela 1 para a região de campos.

Tabela 1: Valores utilizados no cálculo da emissão dos gases.

\begin{tabular}{lc}
\hline Tipo de Vegetação & Região de Campos \\
Densidade de Biomassa (t/ha) & 7,20 \\
Eficiência de queima & 0,95 \\
Fração de Biomassa Viva (FBV) & 0,27 \\
FBV oxidada & 0,81 \\
Carbono da Biomassa Viva & 0,45 \\
Carbono Biomassa Morta & 0,40 \\
\hline
\end{tabular}

Fonte: MCE, 2002.

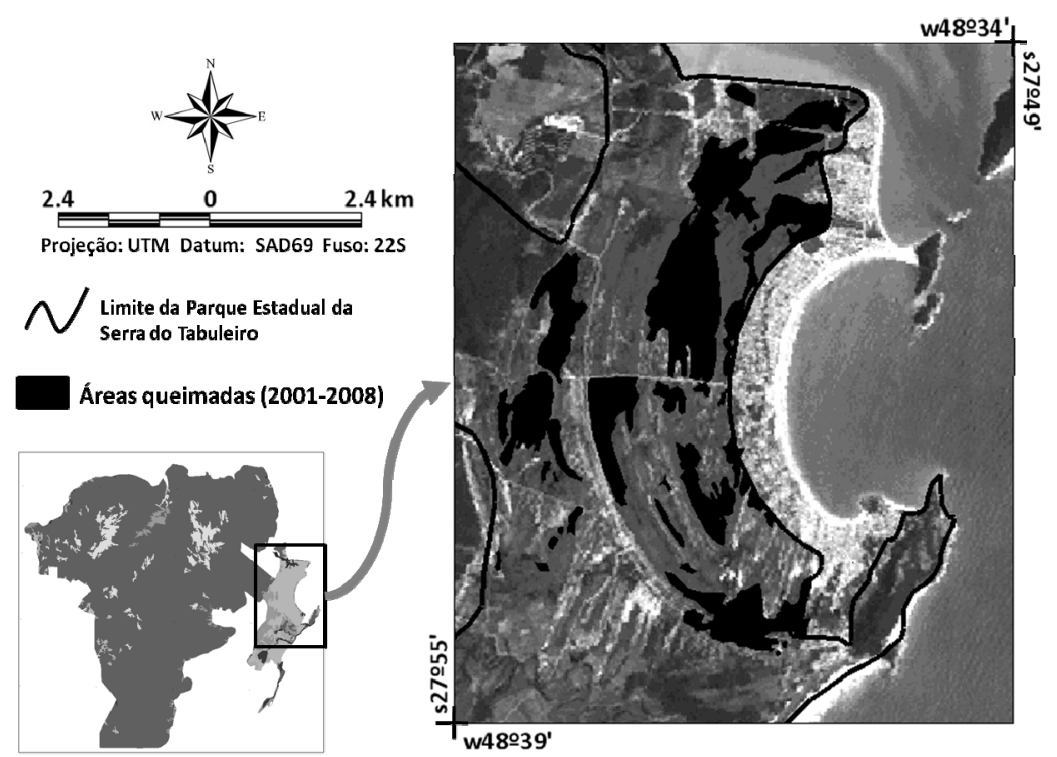

Figura 5n: Imagem da área queimada total (2001 a 2008). 
PEREIRA, G. et al. Análise das áreas queimadas e das emissões dos gases ...

Os resultados da emissão total de gases por compostos para os 1375 ha de biomassa exposta à queima foram de aproximadamente $20 \mathrm{t}$ de $\mathrm{CH}_{4} ; 13.000 \mathrm{t}$ de $\mathrm{CO}_{2} ; 525 \mathrm{t}$ de $\mathrm{CO} ; 7 \mathrm{t}$ de $\mathrm{N}_{2} \mathrm{O}$ e 250 t de $\mathrm{NO}_{\mathrm{x}}\left(\mathrm{NO}+\mathrm{NO}_{2}\right)$. Estas estimativas mostram que as emissões de queimadas provocaram substanciais mudanças na composição química local durante o período de queima, principalmente em relação à emissão dos gases do efeito estufa. Embora boa parte dos compostos emitidos sejam novamente absorvidos para originar novas formações vegetais na região queimada, as sucessivas queimas de biomassa expõem o solo a processos exaustivos, acarretando a menor absorção de carbono na estruturação da nova vegetação (ICHOKU; KAUFMAN, 2005).

Outro impacto ambiental ocasionado pelas queimadas é a alteração no balanço radiativo, que ocorre principalmente devido à mudança nos valores de albedo e das reflectâncias no visível e infravermelho solar. As reduções destes valores propiciam uma maior absorção de radiação eletromagnética (REM) pela superfície, que influencia diretamente o fluxo de calor latente e sensível, a temperatura, a evapotranspiração, o regime de precipitação local, entre outros.

A Figura 6 mostra os valores de albedo e das reflectâncias das áreas situadas entre o perfil A-B (Figura 1 no sentido nornordeste para su-sodoeste, 0 a 4099 metros). Estes valores incluem áreas com vegetação intacta e queimadas. Verificou-se que na área recentemente queimada os valores de albedo (cinza escuro) passaram de aproximadamente $12 \%$ para valores entre $2 \%$ a $6 \%$, uma diminuição de $83 \%$. Em relação à reflectância na faixa do infravermelho solar (cinza claro) verificou-se que seus valores variavam de $18 \%$ a $20 \%$ em áreas adjacentes à área queimada, e nestas houve uma redução de $80 \%$ de seu valor, ou seja, a reflectância no infravermelho solar variou de $4 \%$ a $8 \%$ dependendo da intensidade da área queimada. A reflectância na faixa do visível (em pontilhado) passou de $3 \%$ para valores abaixo de $1,5 \%$, ou seja, observou-se uma redução de $50 \%$. 
PEREIRA, G. et al. Análise das áreas queimadas e das emissões dos gases ...

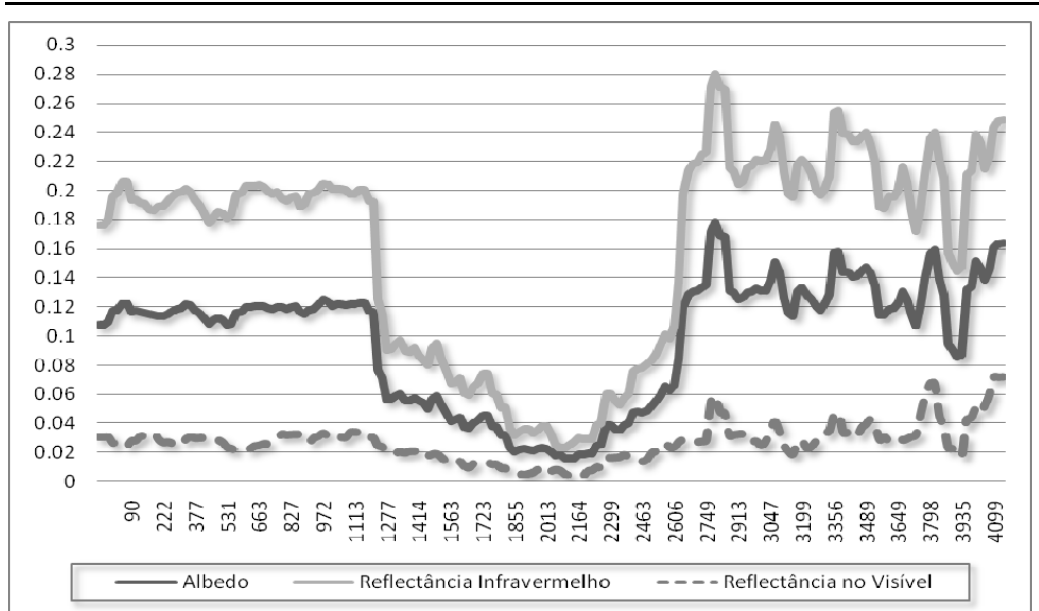

Figura 6: Gráfico de albedo, reflectância no visível e no infravermelho para o dia 21/11/2005 no perfil A-B.

Desta forma, verifica-se que nas áreas queimadas localizadas na região da praia da Pinheira, originam-se áreas de baixa reflectância em todo o espectro eletromagnético devido ao tipo de cobertura do solo. Como verificado em campo, as áreas queimadas encontram-se em áreas suscetíveis a alagamentos, deste modo, ao se remover a cobertura vegetal, a resposta espectral da água passa a dominar o sinal coletado pelo sensor, abaixando ainda mais os valores de albedo e das reflectâncias no visível e no infravermelho.

\section{Considerações finais}

O impacto ambiental das queimadas é um tema preocupante, pois envolve a fertilidade dos solos, a destruição da biodiversidade, a fragilização de ecossistemas, a produção de gases do efeito estuda e nocivos à saúde humana, entre outros. As queimadas interferem diretamente na qualidade do ar, nas características físico-químicas e biológicas dos solos, no balanço radiativo, na vegetação atingida pelo fogo, na fauna local e indiretamente podem afetar os recursos hídricos. 
PEREIRA, G. et al. Análise das áreas queimadas e das emissões dos gases ...

Outro grande impacto das queimadas no meio ambiente é a extinção de espécies nativas, com grandes prejuízos à biodiversidade. Entre a flora presente em todo o parque, há 91 famílias botânicas, com aproximadamente 2.250 espécies. Dentre elas, há 27 endêmicas, raras ou ameaçadas de extinção, destacando-se a presença da espécie conhecida como cavalinha (Equisetum giganteum), que segundo Rosário (2003), “é considerada como fóssil vivo, sendo um dos representantes dos grupos vegetais mais antigos, com 350 milhões de anos".

No Parque Estadual da Serra do Tabuleiro os animais de hábitos terrestres ou aquáticos se abrigam e se reproduzem nos mais variados ambientes úmidos da região. Alguns animais encontrados no parque e que estão ameaçados de extinção são a jacutinga (Papile jacutinga), o bugio (Alouatta fusca), entre outros (ROSÁRIO, 2003).

Assim, pode-se dizer que os resultados obtidos para o período de 2001 a 2008 são preocupantes, pois além da queima de biomassa estar contribuindo com a emissão de gases do efeito estufa, também destrói a biodiversidade do parque, característica importante para atividades científicas, educativas e turísticas ali presente.

\section{Referências bibliográficas}

AMBIENTE BRASIL. http://www.ambientebrasil.com.br/ composer.php3?base $=. /$ snuc/index.html\& conteudo $=. / \mathrm{snuc} / \mathrm{sul} / \mathrm{pe} / \mathrm{ta}$ buleiro.html), acesso 18 de novembro de 2007.

ANDREAE, M. O. Biomass Burning: its history, use, and distribution and its impact on environmental quality and global climate. In: Levine, J. S. (Ed.). Global Biomass Burning: Atmospheric, Climatic, and Biospheric Implications, The MIT Press, Cambridge, MA, p. 2-21, 1991.

BADARINATH, K. V.S.; LATHA, K. M.; CHAND, T. R. K.; GUPTA, P. K.; GHOSK, A. B.; JAIN, S. L.; GERA, B. S.; SINGH, R.; SARKAR, A. K.; SINGH, N.; PARMAR, R. S.; 
PEREIRA, G. et al. Análise das áreas queimadas e das emissões dos gases ...

KOUL, S.; KOHLI, R.; NATH, S.; OJHA, V. K. SIGH, G. Characterization of aerosols from biomass burning - a case study from Mizoram (Northeast), India. Chemosphere, n. 54, p. 167175, 2004.

BERNER, R. A.; BEERLING, D. J.; DUDLEY, R; ROBINSON, J. M.; WILDMAN JR., R. A. Phanerozoic atmospheric oxygen. Annu. Rev. Earth Planet. Sci., n. 31, p. 105-134, 2003.

CHANDER, G.; MARKHAM, B. Revised Landsat-5 TM Radiometric Calibration Procedures and Postcalibration Dynamic Ranges. IEEE Trans. Geosc. And Remote Sens. $41<11>$ : 26742677, 2003.

FINKELSTEIN, D. B.; PRATT, L. M.; BRASSELL, S. C. Can Biomass Burning produce a globally significant carbon-isotope excursion in the sedimentary record? Earth and Planetary Science Letters, doi:10.1016/j.epsl.2006.08.010, 2006.

FINKELSTEIN, D. B. Thoughts on fires. Palaios, n. 19, p. 111112,2004

GLASSPOOL, I. J.; EDWARDS, D.; AXE, L. Charcoal in the Silurian as evidence of the earliest wildfires. Ecology, n. 32, p. 381 $-383,2004$.

GLCF (Global Land Cover Facility). Geocover Technical Guide. Produced by University of Maryland/USA. Disponível em: $<$ http://glcf.umiacs.umd.edu/data/guide/>. Acesso em: 10/09/2006.

IPCC, OECD, IEA. Revised 1996 IPCC Guidelines for National Greenhouse Gas Inventories: Workbook. Bracknell, UK, 2000.

ICHOKU, C.; KAUFMAN, Y. J. A method to derive smoke emission rates from MODIS fire radiative energy measurements. IEEE Trans. on Geosc. \& Rem. Sens., v. 43, n. 11, p. 2636-2649, 2005. 
PEREIRA, G. et al. Análise das áreas queimadas e das emissões dos gases ...

KILLLOPS, S. D.; MASSOUD, M. S. polycyclic aromatic hydrocarbons of pyrolytic origin in acient sediments: evidence for Jurassic vegetation fires. Org. Geochem., n. 18, p 1-7, 1992.

LEVINE, J. S. Biomass burning and the production of greenhouse gases. In: Zepp, R. G. (Ed.) Climate Biosphere Interaction: Biogenic Emissions and Environmental Effects of Climate Change. ISBN 0-471-58943-3, 1994. Disponível em: http://asdwww.larc.nasa.gov/biomass_burn/biomass.html. Acesso em: 24 jan. 2007.

LIANG, S., Narrowband to broadband conversions of land surface albedo I algorithms. Remote Sensing of Environment, v. 76, p. 213-238, 2000.

MAIA, Fabiana Britto de Azevedo. Análise do Turismo em Relação ao Uso Público do Parque Estadual da Serra do Tabuleiro no Município de Santo Amaro da Imperatriz, SC. Florianópolis, 2006. 1 v. Dissertação (Mestrado) - Universidade Federal de Santa Catarina.

MCT. First Brazilian Inventory of anthropogenic greenhouse gas emissions background reports. Ministry of Science and Technology, 2002. Disponível em: < http://ftp.mct.gov.br/Clima/comunic_old/pdf/queimac_i.pdf $>$.

Acesso em: 1 set. 2006.

OLIVEIRA, Ivone Adelina de. Gestão de Conflitos em Parques: estudo de caso do entorno nordeste do Parque Estadual da Serra do Tabuleiro - Praia da Pinheira - SC. Florianópolis, 2005. 269 f. Tese (Doutorado) - Universidade Federal de Santa Catarina.

PEREIRA, G.; MORAES, E. C.; ARAI, E.; OLIVEIRA, L. G. L. de. Estudo preliminar da estimativa da reflectância e albedo de microssistemas pantaneiros a partir de imagens de satélite. RBC. Revista Brasileira de Cartografia, v. 59, p. 55-61, 2007. 
PEREIRA, G. et al. Análise das áreas queimadas e das emissões dos gases ...

ROSÁRIO, L. A. do. Paisagem e Conservação. In: FATMA. A Natureza do Parque Estadual da Serra do Tabuleiro. Florianópolis: FATMA, 2003.

SCOTT, A. C. The pre-Quaternary history of fire. Palaeogeogr. Palaeoclimatol. Palaeocol., n. 164, p. 281-329, 2000.

SEILER, W.; CRUTZEN, P. J. Estimates of gross and net fluxes of carbon between the biosphere and the atmosphere from biomas burning. Clim. Change, v. 2, p. 207-248, 1980.

VENKATESAN, M. J.; DAHL, J. Organic geochemical evidence for global fires at the Cretaceous/Tertiary bondary. Nature, n. 338, p. 57-60, 1989.

VERMOTE, E. F; TANRE, D.; DEUZE, J. L.; HERMAN, M.; MORCRETTE J. J. Second Simulation of the satellite signal in the solar spectrum, 6S: An overview. IEEE Trans. Geosc. And Remote Sens. $35<3>$ : 675-686, 1997.

Recebido em novembro de 2007

Aceito em outubro de 2008 